\title{
IDOSOS RESIDENTES NO MEIO URBANO E SUA REDE DE SUPORTE FAMILIAR E SOCIAL ${ }^{1}$ ELDERLY URBAN RESIDENTS AND THEIR FAMILY AND SOCIAL SUPPORT NETWORKS RED DE APOYO FAMILIAR Y SOCIAL DE ANCIANOS RESIDENTES EN EL MEDIO URBANO
}

\author{
Marinês Tambara Leite², Iara Denise Endruweit Battisti , Evelise Morais Berlezi ${ }^{4}$, Ângela Inês Scheuer ${ }^{5}$
}

\footnotetext{
${ }^{1}$ Texto elaborado a partir do Trabalho de Conclusão do Curso de Enfermagem da Universidade Regional do Noroeste do Estado do Rio Grande do Sul (UNIJUÍ).

${ }^{2}$ Doutora em Gerontologia Biomédica. Docente do Departamento de Ciências da Saúde da UNIJUÍ. Rio Grande do Sul, Brasil.

${ }^{3}$ Mestre em Estatística. Docente do Departamento de Física, Estatística e Matemática da UNIJUÍ. Rio Grande do Sul, Brasil.

${ }^{4}$ Mestre em Gerontologia Biomédica. Docente do Departamento de Ciências da Saúde da UNIJUÍ. Rio Grande do Sul, Brasil.

${ }^{5}$ Enfermeira da Secretaria Municipal de Saúde de Alecrim. Rio Grande do Sul, Brasil.
}

PALAVRAS-CHAVE: Idoso. Família. Geriatria.
RESUMO: Este estudo tem o objetivo de analisar o suporte familiar e social de idosos residentes no município de Alecrim - RS. Participaram do mesmo 338 idosos, sendo a coleta de dados realizada nos meses de março e abril de 2007, utilizando-se dois instrumentos, um com questões sóciodemográficas e outro o Medical Outcomes Stud. Realizou-se análise estatística descritiva e teste de Kruskal-Wallis, Mann-Whitney e qui-quadrado no nível de 5\% de significância. Os resultados mostraram predomínio do sexo feminino (61,8\%), casados (61,5\%), na faixa etária de 60 a $69(56,2 \%)$, coabitando com cônjuge $(49,7 \%)$, tem amigos $(99,1 \%)$, participam de grupos $(35,8 \%)$. Também mostram que um significativo percentual $(87 \%)$ dos idosos possui uma rede familiar e social que lhes fornecem suporte de natureza emocional, material, afetiva e informativa, caracterizando uma interação social positiva. Conclui-se que estes idosos têm bom convívio familiar e social, o que lhes possibilita viver dignamente e com qualidade de vida.

KEYWORDS: Aged. Family. Geriatrics.

ABSTRACT: The objective of this study was to analyze family and social support given to elderly people in Alecrim City, RS, Brazil. Using an analytical and quantitative approach 338 elderly people responded to socio-demographic questions in March and April of 2007. The other instrument used for data collection was the Medical Outcomes Study. We applied descriptive statistical analysis, KruskalWallis and Mann-Whitney tests, and the chi-squared test at $5 \%$ significance levels. Results indicate the prevalence of the female gender $(61,8 \%)$, married $(61,5 \%)$, between the ages 60 and $69(56,2 \%)$, living with a partner $(49,7 \%)$, have friends $(99,1 \%)$, and are part of groups $(35,8 \%)$. Data show that among the elderly who were part of this study, a significant percentage $(87 \%)$ have a family and social network, which supplies them emotional, material, affective, and informational support, characterizing positive social interaction. We conclude that these people have a good social and family life, which allows them to live with dignity and quality of life.

PALABRAS CLAVE: Anciano. Familia. Geriatría.

RESUMEN: Investigación de carácter cuantitativo, analítico, que tuvo por objetivo analizar el apoyo familiar y social de mayores residentes en la municipalidad de Alecrim, RS, Brasil. Del cual participaron 338 adultos mayores, siendo que la misma ocurrid los meses de marzo y abril de 2007. Fueron utilizados dos instrumentos para la recolección de datos; uno con cuestiones socios demográficos y el Medical Outcomes Study. Se realizó análisis estadística descriptiva y test de Kruskal-Wallis y Mann-Whitney y test de qui-cuadrado al nivel de 5\% de significación. Los resultados mostrarán preponderancia de mayores del sexo femenino, unos $(61,8 \%)$, con parejas, $(61,5 \%)$, en la franja de edad entre 60 y 69 años, $(56,2 \%)$, conviviendo con la pareja $(49,7 \%)$, que tiene amigos, $(99,1 \%)$, y, por fin, los que participan de grupos, $(35,8 \%)$. Los datos indicaron que entre los mayores analizados, hay un significativo porcentaje $(87 \%)$ de aquéllos que poseen una red familiar y social que les da soporte de naturaleza emocional, material, afectiva e informativa, caracterizando, así, una interacción social positiva. Se concluye que estas personas mayores tienen una buena convivencia familiar y social, lo que les posibilita vivir con dignidad y con calidad de vida.
Marinês Tambara Leite

Endereço: Rua Floriano Peixoto, 776

98.700-000 - Centro, Ijuí, RS, Brasil.

E-mail: marinesl@unijui.edu.br
Artigo original: Pesquisa Recebido em: 15 de outubro de 2007 Aprovação final: 7 de maio de 2008 


\section{INTRODUÇÃO}

Todo ser humano por sua natureza sempre deseja estar inserido em um determinado meio social, seja no ambiente doméstico, de trabalho ou grupo de amigos. Desse modo, naturalmente, os indivíduos que compartilham estes espaços são, também, os provedores de suporte social. Neste cenário, os indivíduos procuram interagir, conhecer e relacionar-se com outras pessoas, vivenciando momentos de lazer, de trabalho e convívio social.

O grupo familiar e a comunidade são espaços naturais de proteção e inclusão social. Além disso, estas estruturas possibilitam a conservação dos vínculos relacionais e a inclusão em projetos coletivos, permitindo melhoria na qualidade de vida. É no ambiente doméstico, na família, no relacionamento com vizinhos que as pessoas estabelecem relações primárias, as quais constituem a sustentação para o enfrentamento das dificuldades cotidianas. ${ }^{1}$

Estudos mostram que o rompimento de vínculos sociais altera as defesas orgânicas das pessoas, deixando-as mais suscetíveis a doenças. Assim, os laços sociais têm influência no estado de saúde e dispor de uma rede de suporte social, a qual proporciona ajuda aos indivíduos que a ela pertence beneficiam a saúde e o bemestar de todos. ${ }^{2-3}$

Quando se trata do contingente populacional formado por idosos observa-se que estes estão inseridos no meio social de várias formas: integrando a família e mantendo interação com seus vizinhos; participando de grupos de terceira idade, nos quais estreitam vínculos de amizade e afetividade; no ambiente de trabalho, desenvolvendo atividades laborais e convivendo com colegas de ofício ou, se aposentado, mantendo vínculo social com ex-colegas de profissão.

Contudo, verifica-se que uma parcela da população idosa afasta-se de todas as relações sociais e fica restrita ao espaço do lar, situação que favorece para a ocorrência de isolamento e solidão. Há, também, idosos que por não possuírem vínculos familiares e/ou sociais ou por não terem condições físicas, psicológicas e financeiras para ter uma vida independente, passam a residir em instituições asilares, ou hospitais-dia, sendo que nesses locais recebem assistência e formam novos vínculos sociais. ${ }^{2}$

Vale salientar, que a maior parte dos idosos possui independência e autonomia seja residindo sós, em suas casas, ou em companhia de pessoas com as quais mantém laços de parentesco. Alguns, mesmo quando residem com parentes, administram sua aposentadoria, tanto em termos de tempo como financeiramente, podem argumentar sobre o que pensam e o que querem de suas vidas, ou seja, são independentes e têm autonomia. Porém, há aqueles que apresentam dependência parcial, quando necessitam da ajuda de outras pessoas para realizar as atividades da vida diária, e há, ainda, aqueles que são dependentes e sem autonomia, requerendo auxílio para toda e qualquer atividade que lhes dizem respeito. ${ }^{4}$

Nesse cenário, o desafio deve ser no sentido de operacionalizar atenção adequada ao idoso, considerando a amplitude e a gravidade de seus problemas funcionais. Para tanto, se faz necessário a implementação de políticas públicas, inclusive de saúde, passíveis de realizarem-se e condizentes com as autênticas necessidades deste grupo etário. ${ }^{5}$

No contexto familiar, muitos são os sentimentos construídos na convivência entre o idoso e seus familiares. O afeto, a ajuda mútua e a compreensão são aspectos essenciais que devem existir no relacionamento idoso/família. Assim, o convívio se torna agradável e os idosos conseguem viver de forma harmoniosa junto a seus entes queridos. Por outro lado, em muitos casos, identifica-se que tal convivência apresenta turbulências, podendo levar a desentendimentos e desgastes no relacionamento. ${ }^{5}$ Isso pode acontecer por diversos motivos, seja por divergência de idéias ou devido à dependência do idoso em relação a seus íntimos.

A possibilidade atual de convivência simultânea de várias gerações de uma mesma família, faz com que sejam colocadas lado a lado diferentes visões de mundo e de valores, esta condição favorece para o surgimento de conflitos vivenciados entre as gerações no espaço doméstico. ${ }^{6}$ Muitas vezes os velhos, por terem reduzida flexibilidade a mudanças, têm maior dificuldade em se adaptar a essa nova realidade, ou seja, coabitar o mesmo ambiente com pessoas de gerações distintas.

Considerando estes aspectos pressupõese que as pessoas idosas necessitam e possuem alguma forma de suporte social, pois nenhum indivíduo, jovem ou idoso, consegue viver isoladamente de sua família e/ou da sociedade. Além disso, supõe-se que o apoio necessário para a realização das atividades cotidianas e instrumentais da vida diária a idosos no ambiente doméstico é fornecido, principalmente, por pessoas com as quais eles mantêm vínculos. 
O presente estudo teve como objetivos analisar em quais dimensões os idosos necessitam de suporte social e identificar associações entre variáveis sócio-demográficas e situações de suporte social na população de idosos residentes no meio urbano do município de Alecrim, RS.

\section{MATERIAIS E MÉTODOS}

Este estudo caracteriza-se como transversal, quantitativo e analítico, tendo como local de investigação o município de Alecrim, RS.

A população do estudo é constituída por todos os idosos (414) que residem no meio urbano do município de Alecrim, RS. A amostra compõe-se de 338 indivíduos, uma vez que do total, 76 idosos não participaram por uma das seguintes razões: recusar-se a participar do estudo, ter mudado o domicílio para outras cidades, por ter ido a óbito ou porque não se encontravam em seus domicílios por ocasião de três visitas da pesquisadora.

Para integrar o conjunto de participantes do estudo foram estabelecidos como critérios de inclusão: ter idade igual ou superior a 60 anos, ambos os sexos, residir no meio urbano do município de Alecrim e aceitar fazer parte da pesquisa. Foram excluídos da investigação os que não possuíam condições cognitivas e que não verbalizam em português.

Os aspectos éticos de que trata a Resolução No 196/96 do Conselho Nacional de Saúde ${ }^{8}$ foram observados. Assim, o projeto de pesquisa recebeu aprovação do Comitê de Ética em Pesquisa da Universidade Regional do Noroeste do Estado do Rio Grande do Sul, por meio do Parecer Consubstanciado $\mathrm{N}^{\mathrm{o}} 008 / 2007$ e os idosos sujeitos do estudo, assinaram o Termo de Consentimento Livre e Esclarecido (TCLE).

\section{Coleta de dados}

A coleta dos dados foi realizada nos meses de março e abril de 2007, com a utilização de dois instrumentos. Um composto de questões relativas a dados sócio-demográficos e o outro foi o questionário de apoio social aplicado no Medical Outcomes Study (MOS) por Sherbourne, traduzido para o português e validado em estudo realizado com a população do Rio de Janeiro. ${ }^{7}$ Este é composto por 19 itens que abrangem cinco dimensões: de apoio social material; afetiva; interação social positiva; emocional; informação. As opções de resposta para cada item variam de 1 (nunca) a 5 (sempre).

A coleta dos dados foi realizada por uma das pesquisadoras, nos meses de março e abril de 2007, com o auxílio dos Agentes Comunitários de Saúde (ACSs), que forneceram a informação sobre as residências nas quais havia idosos. Ciente deste dado, a pesquisadora realizou visitas a cada uma das residências, momento em que solicitava a colaboração da pessoa idosa para participar do estudo. Caso houvesse aquiescência da mesma, a pesquisadora explanava sobre os objetivos do estudo e ambos assinavam o TCLE.

\section{Análise dos dados}

$\mathrm{Na}$ análise univariada utilizou-se tabelas de freqüências simples com valores absolutos e relativos para analisar as características sóciodemográficas e medidas descritivas para analisar os escores. O valor do escore padronizado para cada uma das cinco dimensões foi obtido pela soma dos pontos obtidos nos itens da dimensão dividido pela pontuação máxima da mesma e, então, multiplicado por cem, sendo que a pontuação de cada item de cada dimensão poderia variar do valor 1 (nunca) até valor 5 (sempre). ${ }^{7}$ Assim, quanto maior o escore, maior será o nível de apoio social. Também foi utilizado o teste de Kruskal-Wallis e Mann-Whitney para comparar os escores entre as dimensões, pois a distribuição dos escores foi muito assimétrica. Na análise bivariada, para avaliar a associação entre as variáveis sóciodemográficas e itens das dimensões utilizou-se o teste de qui-quadrado considerando nível de $5 \%$ de significância. Utilizou-se o software SPSS versão 7.5 para análise estatística.

\section{RESULTADOS}

A Tabela 1 mostra os dados relativos às características sócio-demográficas dos idosos estudados. Verificou-se predomínio do sexo feminino $(61,8 \%)$, estado marital casado ou com união estável $(61,5 \%)$, faixa etária de 60 a 69 anos de idade (56,2\%) e que professam a religião católica $(90,8 \%)$. Identificou-se, ainda, que $49,7 \%$ dos idosos coabitam a residência somente com seu cônjuge, 89,3\% são aposentados e $48,8 \%$ deles possuem renda de um salário mínimo. 
Tabela 1 - Características sócio-demográficas da população urbana de idosos de Alecrim - RS, 2007.

\begin{tabular}{|c|c|c|}
\hline Características & $\mathbf{n}$ & $\%$ \\
\hline \multicolumn{3}{|l|}{ Sexo } \\
\hline Feminino & 209 & 61,8 \\
\hline Masculino & 129 & 38,2 \\
\hline \multicolumn{3}{|l|}{ Estado marital } \\
\hline Casado(a)/união estável & 208 & 61,5 \\
\hline Viúvo/solteiro/separado & 130 & 38,5 \\
\hline \multicolumn{3}{|l|}{ Idade } \\
\hline 60 a 69 anos & 190 & 56,2 \\
\hline 70 a 79 anos & 110 & 32,5 \\
\hline 80 anos ou mais & 38 & 11,2 \\
\hline \multicolumn{3}{|l|}{ Religião } \\
\hline Católica & 307 & 90,8 \\
\hline Outras & 31 & 9,2 \\
\hline \multicolumn{3}{|l|}{ Coabita a residência com } \\
\hline Cônjuge & 168 & 49,7 \\
\hline Cônjuge, filho(a), neto(a) & 48 & 14,2 \\
\hline Filhos e netos & 47 & 13,9 \\
\hline Outros & 10 & 2,9 \\
\hline Pessoa que cuida e outros & 5 & 1,5 \\
\hline Moram só & 60 & 17,8 \\
\hline \multicolumn{3}{|l|}{ Profissão } \\
\hline Aposentado(a) & 302 & 89,3 \\
\hline Do lar & 21 & 6,2 \\
\hline Agricultor(a) & 7 & 2,1 \\
\hline Outros & 8 & 2,4 \\
\hline \multicolumn{3}{|l|}{ Renda (salário mínimo) } \\
\hline 1 & 164 & 48,8 \\
\hline 1,5 & 19 & 5,6 \\
\hline 2 & 106 & 31,6 \\
\hline 2,5 ou + & 39 & 11,6 \\
\hline Sem renda & 8 & 2,4 \\
\hline
\end{tabular}

As médias, respectivos desvios-padrão, as medianas e os valores que limitam os tercis dos escores de apoio social estão apresentados na Tabela 2. Quanto maior o escore, maior o apoio social em cada dimensão, desta forma percebe-se que todas as dimensões tiveram escore médio acima de 80, numa escala que poderia variar de 20 a 100 pon- tos, demonstrando um bom nível de apoio social dos idosos do meio urbano no município. Como a distribuição dos escores de cada dimensão é muito assimétrica, utilizou-se o teste de Kruskal-Wallis para comparar os escores entre as dimensões, verificando-se que pelo menos uma das dimensões difere significativamente $(\mathrm{p}<0,001)$ das demais.

Tabela 2 - Medidas descritivas para os escores das dimensões de apoio social e idosos do meio urbano de Alecrim - RS, 2007.

\begin{tabular}{lccccc}
\hline Dimensão & $\mathbf{N}^{\circ}$ itens & Média & Desvio-padrão & Mediana & Tercis \\
\hline Material & 4 & 82,95 & 22,798 & 95 & $\geq 20$ e $\leq 80 ; \geq 85$ e $\leq 95 ; 100$ \\
Afetivo & 3 & 87,87 & 16,562 & 100 & $\geq 40$ e $\leq 80 ; \geq 87$ e $\leq 93 ; 100$ \\
Emocional & 4 & 88,55 & 19,190 & 100 & $\geq 25$ e $\leq 95 ; 100$ \\
Informação & 4 & 87,37 & 18,219 & 100 & $\geq 20$ e $\leq 80 ; \geq 85$ e $\leq 95 ; 100$ \\
Interação positiva & 4 & 89,26 & 16,596 & 100 & $\geq 40$ e $\leq 80 ; \geq 85$ e $\leq 95 ; 100$ \\
\hline
\end{tabular}


Pelo teste de Mann Whitney, observou-se que a dimensão material foi a que teve menores prevalências de respostas quase sempre/sempre, sendo esta a dimensão que diferiu significativamente das demais (Tabela 2). Também, a dimensão informação, diferiu expressivamente da emocional e da interação positiva, sendo que as demais não se diferenciaram significativamente entre si.

A Tabela 3 mostra os valores de $p$ (significância) relativo ao teste de qui-quadrado para a associação da freqüência que o idoso conta com ajuda de alguém, em cada item das dimensões material, afetivo, emocional, informações e interação positiva em relação às características sóciodemográficas. Verificou-se que na dimensão material há associação significativa entre o item "para preparar suas refeições" com sexo, estado marital e renda. Na análise estatística dos itens da dimensão afetiva com as características sócio-demográficas, não houve associação significativa.

Tabela 3 - Associação da freqüência que o idoso conta com ajuda de alguém em cada item das dimensões estudadas, Alecrim - RS, 2007.

\begin{tabular}{|c|c|c|c|c|c|c|}
\hline \multirow[b]{2}{*}{ Item } & \multicolumn{6}{|c|}{ Características sócio-demográficas } \\
\hline & Sexo & Idade & $\begin{array}{l}\text { Estado } \\
\text { marital }\end{array}$ & Renda & $\begin{array}{l}\text { Faz } \\
\text { visita }\end{array}$ & $\begin{array}{c}\text { Grupo } \\
3^{\mathrm{a}} \text { idade }\end{array}$ \\
\hline \multicolumn{7}{|l|}{ Dimensão material } \\
\hline Se ficar na cama & 0,350 & 0,363 & 0,450 & 1,000 & 0,521 & 0,096 \\
\hline Para levá-lo ao médico & 0,588 & 0,298 & 1,000 & 1,000 & 1,000 & 0,528 \\
\hline Para ajudá-lo nas tarefas diárias & 0,104 & 0,553 & 0,256 & 0,524 & 0,377 & 0,737 \\
\hline Para preparar suas refeições & $<0,001$ & 0,670 & 0,007 & 0,026 & 0,408 & 0,690 \\
\hline \multicolumn{7}{|l|}{ Dimensão afetiva } \\
\hline Que demonstre amor e afeto por você & 0,320 & 0,387 & 0,130 & 0,324 & 0,164 & 0,238 \\
\hline Que lhe dê um abraço & 0,320 & 0,387 & 0,130 & 0,324 & 0,164 & 0,238 \\
\hline Que você ame e que faça você se sentir querido & 0,320 & 0,387 & 0,130 & 0,324 & 0,164 & 0,238 \\
\hline \multicolumn{7}{|l|}{ Dimensão emocional } \\
\hline Para ouvi-lo, quando você precisar falar & 0,155 & 0,052 & 0,032 & 0,162 & 0,027 & 0,032 \\
\hline $\begin{array}{l}\text { Em quem confiar ou para falar de você ou sobre seus } \\
\text { problemas }\end{array}$ & 0,191 & 0,137 & 0,010 & 0,127 & 0,030 & 0,025 \\
\hline Para compartilhar suas preocupações medos mais íntimos & 0,204 & 0,132 & 0,009 & 0,134 & 0,030 & 0,026 \\
\hline Que compreenda seus problemas & 0,162 & 0,156 & 0,027 & 0,204 & 0,013 & 0,011 \\
\hline \multicolumn{7}{|l|}{ Dimensão informação } \\
\hline Para dar bons conselhos em situações de crise & 0,689 & 0,185 & 0,158 & 0,189 & 0,074 & 0,121 \\
\hline $\begin{array}{l}\text { Para dar informação que o ajude a compreender uma } \\
\text { determinada situação }\end{array}$ & 0,038 & 0,004 & 0,326 & 0,204 & 0,020 & 0,038 \\
\hline De quem você realmente quer conselhos & 0,106 & 0,045 & 0,119 & 0,486 & 0,020 & 0,051 \\
\hline Para dar sugestões de como lidar com um problema & 0,044 & 0,071 & 0,863 & 0,554 & 0,025 & 0,017 \\
\hline \multicolumn{7}{|l|}{ Dimensão interação positiva } \\
\hline Com quem fazer coisas agradáveis & 0,973 & 0,026 & 0,040 & 0,548 & 0,062 & 0,001 \\
\hline Com quem distrair a cabeça & 0,973 & 0,026 & 0,040 & 0,548 & 0,062 & 0,001 \\
\hline Com quem relaxar & 0,973 & 0,026 & 0,040 & 0,548 & 0,062 & 0,001 \\
\hline Para se divertir junto & 0,973 & 0,026 & 0,040 & 0,548 & 0,062 & 0,001 \\
\hline
\end{tabular}

*Valor p correspondente ao teste de qui-quadrado com correção de continuidade para sexo, situação conjugal, renda, faz visita, grupo $3^{\mathrm{a}}$ idade e teste de qui-quadrado para idade; categorias de renda: $1 \mathrm{SM}$ e > 1SM; categorias de faz visita (sim e não); categorias de participa de grupo de terceira idade (sim e não);

** Freqüência com que o idoso conta com alguém categorizado em nunca/raramente/às vezes e quase sempre/sempre. 
Já, quanto aos itens da dimensão emocional constatou-se associação estatisticamente significativa na relação entre o item "para ouvi-lo quando você precisa falar" com idade, estado marital, a condição de fazer visita e também com a condição de fazer parte de grupo de terceira idade. Também, houve associação entre "em quem confiar ou para falar de você ou sobre seus problemas" com estado marital, condição de fazer visita e de participar de grupo de terceira idade. Para estas mesmas variáveis sócio-demográficas, houve associação significativa com os itens "para compartilhar suas preocupações e medos mais íntimos" e "que compreenda os seus problemas".

Na dimensão informação verificou-se associação significativa entre os itens "para dar informações que o ajude a compreender uma determinada situação" com sexo, idade, fazer visitas e participar de grupo de terceira idade; o item "de quem você realmente quer conselhos" associou-se com as mesmas variáveis, exceto com sexo. E, no item "para dar sugestões de como lidar com um problema" verificou-se associação significativa com sexo, condição de fazer visitas e de participar de grupo de terceira idade.

Quando realizada a análise estatística entre os itens da dimensão interação positiva (com quem fazer coisas agradáveis, com quem distrair a cabeça, com quem relaxar e para se divertir junto) constatou-se que todos os itens desta dimensão associam-se com as variáveis idade, estado marital, condição de fazer visita e de participar de grupo de terceira idade.

\section{DISCUSSÃO}

Referentes à caracterização social e demográfica, os dados encontrados no presente estudo são semelhantes aos padrões brasileiros, pois quando se trata da população idosa, há predominância de mulheres e prepondera a união estável, na faixa etária de 60 a 69 anos de idade, que seguem a religião católica, residem com cônjuge/companheiro, a maior parte é aposentado e percebem até um salário mínimo de renda, como aponta os dados do Censo Demográfico ${ }^{9}$ de 2000.

Estudo sobre a dependência de idosos, aponta que cerca de $40 \%$ dos indivíduos com 65 anos ou mais de idade precisam de algum tipo de ajuda para realizar pelo menos uma tarefa instrumental da vida diária como: fazer compras, cuidar das finanças, preparar refeições e limpar a casa. Enfatiza que a transição demográfica e a transição epidemio- lógica apresentam, cada vez mais, um quadro de sobrevivência de idosos na dependência de uma ou mais pessoas que suprem as suas incapacidades para a realização das atividades de vida diária. ${ }^{10}$

O cotidiano dos indivíduos é permeado de situações, alegres ou não, e comumente eles desejam e necessitam socializar com outra pessoa. Assim, é importante poder contar com alguém que se disponha a escutar, dialogar e, muitas vezes, ajudar a compreender, solucionar ou mesmo aceitar os acontecimentos diários. Em se tratando de indivíduos que estão vivenciando a velhice, a necessidade de socializar os fatos corriqueiros da vida, também, faz parte de sua existência. O compartilhamento de emoções e sentimentos, freqüentemente, ocorre entre familiares e amigos. Entretanto, muitos idosos não possuem filhos ou familiares próximos e somente contam com os amigos para interagir e dialogar, constituindo-se em uma rede de apoio.

No que tange a vínculos interpessoais, identifica-se na literatura sobre apoio e rede social, que os gerontes devem manter as relações sociais com familiares e, principalmente, com amigos da mesma geração, pois favorece o bem-estar psicológico e social dos idosos. Os relacionamentos entre amigos idosos são particularmente benéficos, porque são de livre escolha e mais funcionais ao atendimento das necessidades afetivas dos envolvidos. ${ }^{11}$

Estudo sobre características sócio-demográficas, suporte social e indicadores de condição de saúde dos idosos ${ }^{12}$ evidenciou a existência de importante rede de solidariedade entre gerações, identificada por meio da ajuda que o idoso recebe (financeira, moradia e/ou companhia) e presta à sua família. Em relação às condições de saúde, a pesquisa concluiu que todos os indicadores utilizados mostraram agravamento do estado de saúde com o aumento da idade. Este fato indica que os idosos mais velhos passam a ter mais necessidade de auxílio de outra pessoa. Nessa condição, a existência de uma rede de suporte, familiar ou social, torna-se extremamente relevante.

A construção da rede social se faz ao longo da vida, porém também pode constituir-se em qualquer etapa do ciclo vital. Atualmente, em especial, entre os idosos, uma das formas de congregar e assistir este estrato populacional, é por meio dos grupos de terceira idade, os quais se constituem em espaços de socialização, interação e valorização, atributos que favorecem a independência e autonomia e possibilitam a manutenção do exercício da cidadania. ${ }^{13}$ 
A interação social vivenciada pelos idosos colabora para o exercício de sua cidadania e, também, para que o indivíduo se sinta valorizado e inserido no meio social. Além disso, o idoso passa a ter a sensação de pertencimento a um grupo social, para o qual pode contribuir de maneira significativa utilizando-se de sua experiência e conhecimento. No entanto, deve-se ter claro que a interação social não ocorre somente em espaços públicos, mas em qualquer ambiente, seja familiar, igreja, escola, enfim em todo local em que há respeito e valorização pelo ser humano, em especial, a pessoa idosa.

O incentivo dos filhos e da família para que os idosos participem de grupos de terceira idade é fundamental, uma vez que por meio da socialização há possibilidade de redefinir papéis tais como: trabalho, identidade, solidariedade, participação social, convivência, entre outros. ${ }^{14}$

Nem todos os idosos podem dispor do cuidado de um familiar, pois existem alguns que não têm familiares com laços de parentesco em primeiro ou segundo grau, uma vez que não contraíram matrimônio, outros por serem viúvos ou por não terem tido filhos. O fato de morar só, para o idoso, tem sido associado a um decréscimo na qualidade de vida, agravamento de morbidades e, até mesmo, indicador de risco de mortalidade. ${ }^{15}$ Nessas situações, cabe à sociedade fornecer o apoio necessário a esses indivíduos por meio de programas e serviços que possam auxiliá-los no restabelecimento da saúde e contribuir para a reestruturação da sua qualidade de vida.

A tendência, hoje existente, de os idosos morarem sozinhos não tem de ser, necessariamente, entendida como um reflexo de abandono por parte de seus familiares. Ela pode significar um novo tipo de arranjo, uma nova forma de a família viver. ${ }^{16}$

Contudo, é necessário estar atento para as questões do envelhecimento, em especial para a possibilidade de os idosos vivenciarem de forma solitária ou sentir-se solitário, mesmo estando residindo ou na companhia de algum familiar. Para alguns, o enfrentamento dessa situação ocorre de maneira tranqüila já, para outros, é motivo de angústia, medo e preocupação, pois não se sentem bem morando sós e desejariam conviver com alguém da família. Nesses casos os familiares devem ficar em posição de alerta e observando as condições psicológicas e de vida desses idosos, identificando e fornecendo a ajuda necessária.

Estudo ${ }^{15}$ referindo-se a provedores de cuidado à pessoa idosa menciona que em situações de adoecimento, hospitalização ou para a realização de atividades diárias, os filhos são os principais apoiadores e cuidadores. Por outro lado, nas situações em que o suporte familiar não está estruturado, o cuidado é prestado por um sistema de suporte informal, ou seja, é exercido por familiares, amigos, vizinhos e membros da comunidade.

É importante para as pessoas com 60 anos ou mais de idade, tanto àquelas que vivem sós quanto as que estão próximas de seus familiares, desempenharem papéis sociais diante de sua família e amigos, para que possam vivenciar a velhice de modo saudável, redefinindo o seu papel social e cultural perante a sociedade. Convivendo direta ou indiretamente com suas referências familiares, o idoso preserva a sua dignidade e assegura-se da manutenção de seu papel existencial. ${ }^{17}$

\section{CONCLUSÕES}

Este estudo alcançou os objetivos propostos e permitiu identificar que dentre os idosos participantes da pesquisa há predomínio do sexo feminino, residem com companheiro, professam a religião católica e podem ser considerados idosos "jovens".

Os dados mostram elevado percentual de idosos que possuem rede familiar e social, a qual pode fornecer suporte de natureza emocional, material, afetiva e informativa, caracterizando interação social positiva. Sendo assim, estes idosos demonstram ter bom convívio familiar e social, o que lhes possibilita viver dignamente e com qualidade de vida. Além disso, o geronte ao sentir-se inserido no grupo familiar e social, poderá contribuir com a sociedade desenvolvendo atividades laborais ou de modo voluntário, de tal modo que suas potencialidades se sobrepõem às suas limitações.

Considera-se que este estudo contém informações que podem auxiliar no planejamento de políticas de atenção a população idosa, especialmente àquela que não dispõe de uma rede de suporte familiar ou social. Também, esperase que, somado a outros, contribua para que os indivíduos possam ter condições de envelhecer, mantendo relacionamento interpessoal, tendo oportunidade de se manterem ativos e de se sentirem bem em relação a si mesmos.

\section{REFERÊNCIAS}

1 Lemos N, Medeiros SL. Suporte social ao idoso dependente In: Freitas EV, Py L, Neri AL, Cançado HAX, Gonzoni ML, Rocha SM. Tratado de geriatria e 
gerontologia. Rio de Janeiro (RJ): Guanabara Koogan; 2002. cap.107, p.892-7.

2 Avlund K, Damsgaard MT, Holstein BE. Social relations and mortality in eleven year follow-up study of 70-year-old men and women in Denmark. Soc. Sci. Med. 1998 September; 47 (5): 635-43.

3 Fratiglione L, Wang HX, Ericsson K, Maytan M, Winblad B. Influence of social network on occurrence of dementia: a community-based longitudinal study. Lancet. 2000 Apr; 355 (15): 1315-9.

4 Papaléo Netto M. O estudo da velhice: histórico, definição do campo e termos básicos. In: Freitas EV, Py L, Cançado FAX, Doll J, Gorzoni ML. Tratado de Geriatria e Gerontologia. Rio de Janeiro (RJ): Guanabara Koogan; 2006. cap. 01, p.2-12.

5 Martins JJ, Albuquerque GL, Nascimento ERP, Barra DCC, Souza WGA, Pacheco WNS. Necessidades de educação em saúde dos cuidadores de pessoas idosas no domicílio. Texto Contexto Enferm. 2007 Abr-Jun; 16 (2): 254-62.

6 Goldim JR. Bioética e envelhecimento. In: Freitas EV, Py L, Neri AL, Cançado HAX, Gonzoni ML, Rocha SM. Tratado de geriatria e gerontologia. Rio de Janeiro (RJ): Guanabara Koogan, 2002. p.85-90.

7 Andrade CR. Associação entre apoio social e freqüência relatada de auto-exame das mamas no estudo pró-saúde [dissertação]. Rio de Janeiro (RJ): Escola Nacional de Saúde/Fundação Oswaldo Cruz; 2001.

8 Ministério da Saúde (BR), Conselho Nacional de Saúde, Comissão Nacional de Ética em Pesquisa. Resolução No 196 de 10 de outubro de 1996: diretrizes e normas regulamentadoras de pesquisa envolvendo com seres humanos. Diário Oficial da União, de 16 de outubro de 1996.
9 Instituto Brasileiro de Geografia e Estatística. Relatórios técnicos 2000 [acesso em 2006 Out 10]. Disponível em: www.ence.ibge.gov.br/publicacoes

10 Karsch UM. Idosos dependentes: famílias ecuidadores. Cad. Saúde Pública. 2003 Jun; 19 (3): 861-6.

11 NERI AL. Oque a psicologia tem a oferecer ao estudo e à intervenção no campo do envelhecimento no Brasil, hoje. In: NERI AN, SANCHEZ MY,organizadores. Velhice bem-sucedida. Campinas (SP): Papirus; 2004, p.13-27.

12 Lima e Costa MFF, Guerra HL, Firmo JOA, Uchoa E. Projeto Bambuí: um estudo epidemiológico de características sociodemográficas, suporte social e indicadores de condição de saúde dos idosos em comparação aos adultos jovens. Inf. Epidemiol. Sus 2001 Dez; 10(4): 147-61.

13 Leite MT, Hildebrandt LM, Nasi C, Rocha MS. O velho e as modalidades de atenção no município de Ijuí/RS. Rev. Contexto Saúde. 2005 Jan/Jun-Jul/ Dez; 5 (8/9): 7-16.

14 Christensen RB. A importância da socialização na terceira idade. Santa Rosa (RS): Unijuí/Departamento de Ciências Sociais; 2004.

15 Caldas CP. O idoso em processo de demência: o impacto na família. In: Minayo MCS, Coimbra Júnior CEA, organizadores. Antropologia, saúde e envelhecimento. Rio de Janeiro (RJ): FIOCRUZ; 2003.

16 Debert GG. A reinvenção da velhice. São Paulo (SP): EDUSP/FAPESP; 2004.

17 Miguel CS, Fortes VLF. Idosas de um grupo de terceira idade: as interfaces da relação com suas famílias. Rev. Bras. Ciências Envelhecimento. 2005 Jul-Dez; 2 (2): 74-85. 